\title{
Endometriose Simulando Neoplasia Vesical
}

\author{
Endometriosis Simulating Bladder Cancer
}

\begin{abstract}
Marcos Tobias-Machado, Antonio C. Lopes Neto, Roberto Vaz Juliano, Lucila H. Simardi,
\end{abstract} Caio P. Barbosa, Milton Borrelli, Eric R. Wroclawski

\begin{abstract}
RESUM0
Objetivo: o acometimento do trato urinário pela endometriose é raro e quando ocorre, a bexiga é o órgão mais freqüentemente afetado. Observamos que algumas pacientes têm sido encaminhadas com o diagnóstico clinico de neoplasia vesical. Em geral, a literatura mostra relatos isolados de casos, tornando dificil a padronização de condutas. Tivemos por objetivo apresentar nossa experiência, mostrando os principais aspectos diagnósticos e terapêuticos desta entidade clinica.

Métodos: avaliamos retrospectivamente os casos com diagnóstico de endometriose vesical por meio do arquivo do Departamento de Patologia, fazendo revisão dos dados clínicos de prontuário e convocando as pacientes para seguimento ambulatorial após tratamento.

Resultados: os principais sinais e sintomas apresentados pelas pacientes foram disúria cíclica, massa e dor pélvica crônica. O diagnóstico presuntivo foi realizado mediante ultra-sonografia (USG), tomografia computadorizada (TC) de abdome, cistoscopia e laparoscopia. O diagnóstico definitivo com confirmação anátomo-patológica foi obtido pela ressecção endoscópica em 3 casos e biópsia laparoscópica em 1 caso. As opções terapêuticas foram o tratamento medicamentoso exclusivo e a ressecção da lesão empregando a via endoscópica ou cistectomia parcial, sempre complementados por tratamento clínico adjuvante.

Conclusões: revisamos os principais aspectos clinicos e terapêuticos da endometriose do trato urinário, lembrando que esta representa um importante diagnóstico diferencial de tumor vesical em mulheres jovens na idade reprodutiva.
\end{abstract}

PALAVRAS-CHAVE: Endometriose. Cirurgia endoscópica. Bexiga. Neoplasia.

\section{Introdução}

A endometriose é uma doença multifocal caracterizada pelo crescimento anormal de tecido endometrial fora da cavidade uterina, de progressão rápida e imprevisível. Os principais sítios de lesão são os ovários, ligamentos útero-sacros e redondo e fundo de saco de Douglas. A patogênese desta afecção é desconhecida, mas algumas teorias têm sido propostas. Estas incluem a menstruação retrógrada, disseminação do tecido endometrial durante cirurgia, remanescentes embrionários, metaplasias, metástases linfáticas e hematogênicas ${ }^{1,2}$. O acometimento do trato

Disciplinas de Urologia, Ginecologia e Patologia da Faculdade de Medicina da Fundação do ABC Correspondência:

Marcos Tobias-Machado

Rua Oscar Freire, 1546 apto 53 - Cerqueira Cesar

05409-010 - São Paulo - SP

Tel: (11) 881-8674 urinário é raro e ocorre em 1-2\% dos casos, sendo a bexiga acometida em $80 \%$ das vezes. O primeiro caso de endometriose vesical foi descrito em $1921^{1,2}$. Em 1960, revisando 6.107 casos de endometriose dentro de grandes séries desde 1925, Abeshouse e Abeshouse ${ }^{3}$ encontraram apenas 67 casos de endometriose vesical, que correspondia a apenas $1,1 \%$ dos possiveis sítios de instalação desta patologia. Nessa extensa revisão, foram descritos 6 casos de endometriose renal $(0,1 \%), 15$ de acometimento ureteral $(0,24 \%)$ e 3 em topografia uretral $(0,05 \%)$. O quadro clínico é variado, podendo apresentar-se como hematúria cíclica, dor pélvica e sintomas vesicais irritativos. Os principais diagnósticos diferenciais incluem angiomas, papilomas, lesões inflamatórias inespecíficas como a cistite eosinofilica, cistite intersticial e a cistite cística, malacoplaquia, tuberculose e carcinoma vesical ${ }^{1-5}$. O diagnóstico preciso é necessário para instituirmos a terapêutica apropriada. 
A maior parte da literatura é representada por relatos isolados de casos, o que dificulta uma adequada padronização de condutas. Tivemos por objetivo apresentar nossa experiência no tratamento de 4 pacientes, apresentando nossas impressões no que tange às dificuldades diagnósticas e aos controversos aspectos terapêuticos.

\section{Pacientes e Métodos}

Foi realizada análise retrospectiva dos laudos e das lâminas de pacientes com diagnóstico de endometriose do trato urinário atendidas no Departamento de Patologia da Faculdade de Medicina do ABC. A partir desta identificação, realizamos levantamento do prontuário a fim de identificar a história clínica, especialmente os sinais e sintomas urinários e ginecológicos, os caminhos tomados até que se estabelecesse o diagnóstico, os exames subsidiários realizados e a conduta terapêutica instituída. Todas as pacientes foram convocadas a pelo menos uma entrevista (caso o tratamento proposto tivesse sido completado), ou a consultas trimestrais para seguimento caso ainda estivessem recebendo tratamento. Esta era realizada por médico que não tinha interferência na decisão do tratamento. Desta maneira era possivel interpretar de maneira imparcial os resultados. Após a coleta dos dados, apresentamos as nossas conclusões baseados na resposta ao tratamento e em revisão da literatura.

\section{Resultados}

Uma compilação dos principais achados clínicos e terapêuticos encontrados em nossos 4 casos são resumidos na Tabela 1. Passamos a apresentar os dados mais detalhados de cada paciente:

Tabela 1 - Dados clínicos, terapêuticos e evolução de 4 pacientes com endometriose vesical.

\begin{tabular}{|c|c|c|c|c|c|c|c|c|}
\hline \multirow[t]{3}{*}{ No. } & \multirow[t]{3}{*}{ Idade } & \multirow{3}{*}{$\begin{array}{l}\text { Motivo avaliação } \\
\text { urológica }\end{array}$} & \multirow[t]{3}{*}{ Imagem } & \multirow[t]{3}{*}{ Biópsia inicial } & \multicolumn{2}{|c|}{ Tratamento } & \multirow{3}{*}{$\begin{array}{c}\text { Tempo } \\
\text { seguimento }\end{array}$} & \multirow[t]{3}{*}{ Resultado } \\
\hline & & & & & Cirúrgico & Clínico & & \\
\hline & & & & & \multicolumn{2}{|c|}{ (análogos do GnRH) } & & \\
\hline 1 & 30 & $\begin{array}{l}\text { Sintomas irritativos/ } \\
\text { massa hipogástrio }\end{array}$ & $\begin{array}{l}\text { TC: grande } \\
\text { tumor pélvico }\end{array}$ & Endoscópica: cistite & - & 6 meses & 3 meses & Em avaliação \\
\hline 2 & 40 & $\begin{array}{l}\text { Disúria cíclica } \\
\text { Polaciúria }\end{array}$ & $\begin{array}{l}\text { USG: massa } \\
\text { vesical } 3 \mathrm{~cm}\end{array}$ & Endoscópica: cistite & RTU & 1 ano & 1 ano & s/ alterações \\
\hline 3 & 41 & $\begin{array}{c}\text { Dor pélvica } \\
\text { Hematúria Disúria }\end{array}$ & USG: lesão 2 cm & $\begin{array}{l}\text { Laparoscópica: } \\
\text { endometriose }\end{array}$ & Cistectomia parcial & Após recidiva & 5 anos & $\begin{array}{l}\text { Recidiva após } 4 \text { anos/ } \\
\text { há } 1 \text { ano s/ alterações }\end{array}$ \\
\hline 4 & 35 & $\begin{array}{l}\text { Dor pélvica Disúria } \\
\text { Polaciúria }\end{array}$ & USG: lesão 5 cm & $\begin{array}{l}\text { Endoscópica: } \\
\text { endometriose }\end{array}$ & RTU & 3 meses & 6 meses & s/ alterações \\
\hline
\end{tabular}

Tc = tomografia computadorizada

USG = ultra-sonografia

Paciente 1: Paciente de 30 anos, com dor no hipogástrio, polaciúria e disúria há 2 anos, com piora dos sintomas no periodo menstrual. Negava hematúria. No exame abdominal palpava-se um tumor sólido, irregular, com limites imprecisos, estendendo-se até $7 \mathrm{~cm}$ acima da sínfise púbica. Urinálise com hematúria e leucocitúria. A USG mostrava bexiga de paredes espessadas com tumor projetando-se em sua luz (Figura 1). A TC confirmou a presença de formação heterogênea complexa (áreas císticas e sólidas) na cavidade pélvica sem plano de clivagem com bexiga, corpo uterino, alças intestinais e musculatura anterior (Figura 2). A cistoscopia revelou compressão extrínseca da parede vesical póstero-superior, cujas biópsias iniciais revelaram cistite inespecífica. Uma terceira biópsia realizada com alça de ressecção mostrou endometriose. Optou-se por tratamento clínico com análogos de GnRH, a fim de se obter regressão desta lesão. Atualmente está em tratamento há 3 meses com melhora clínica parcial e sem redução da massa à TC. Receberá mais 3 meses de tratamento antes da reavaliação.

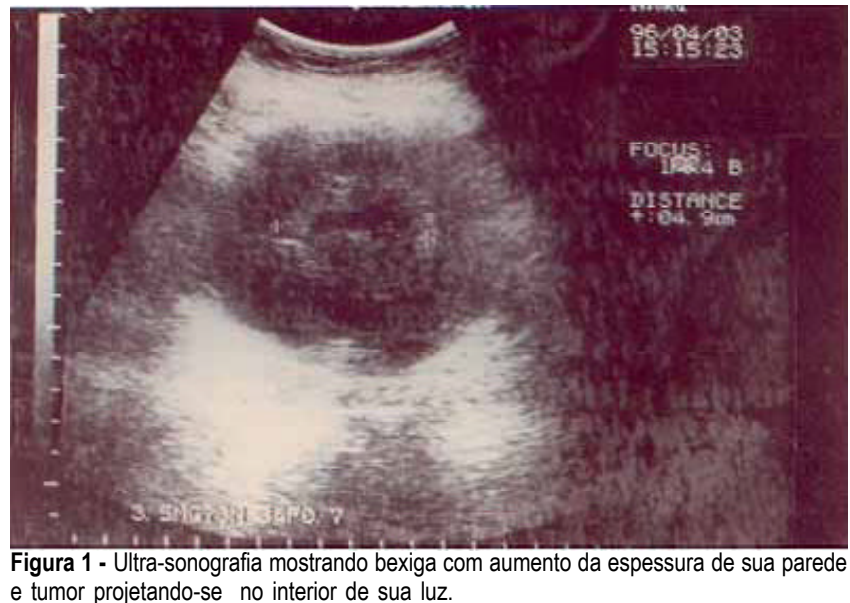




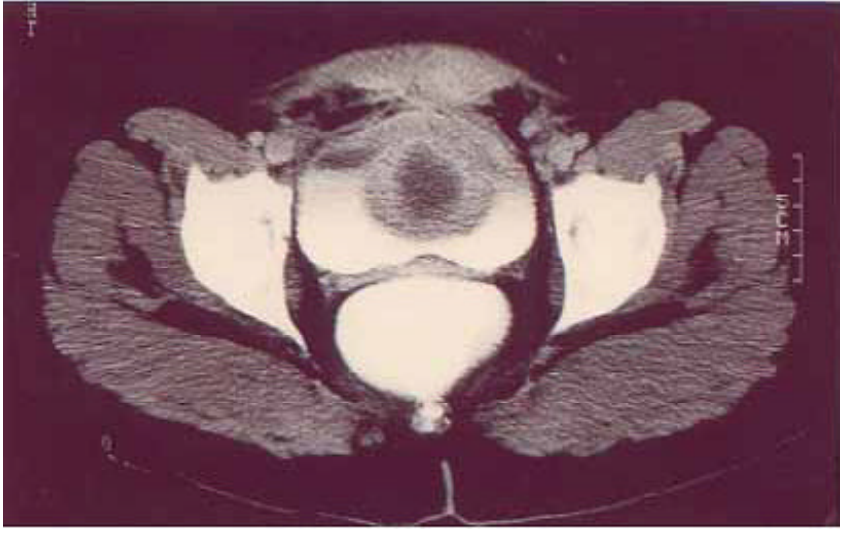

Figura 2 - Tomografia computadorizada da pelve mostrando tumor de grandes dimensões em contigüidade com a bexiga, apresentando limites precisos e área de necrose central.

Paciente 2: Paciente de 40 anos, com episódios de disúria cíclica, polaciúria e hipermenorragia. Negava hematúria ou outros sintomas. Ao exame, apresentava dor à palpação do hipogástrio, sem tumor palpável. O exame ginecológico era normal. Urinálise sem particularidades. A USG mostrou aumento difuso do útero e tumor de $3 \mathrm{~cm}$ em parede lateral da bexiga (Figura 3). A cistoscopia revelou lesão elevada de $3 \mathrm{~cm}$ com mucosa vesical integra e cuja biópsia revelou tratar-se de cistite inespecífica. Durante ressecção transuretral da massa, ocorreu saída de secreção achocolatada da lesão e o exame anátomo-patológico diagnosticou tecido endometrial invadindo a parede muscular da bexiga (Figuras 4A e B). A paciente está em acompanhamento há 1 ano, recebendo tratamento complementar com análogos de GnRH, evoluindo com melhora clínica e ausência de recidiva à USG.

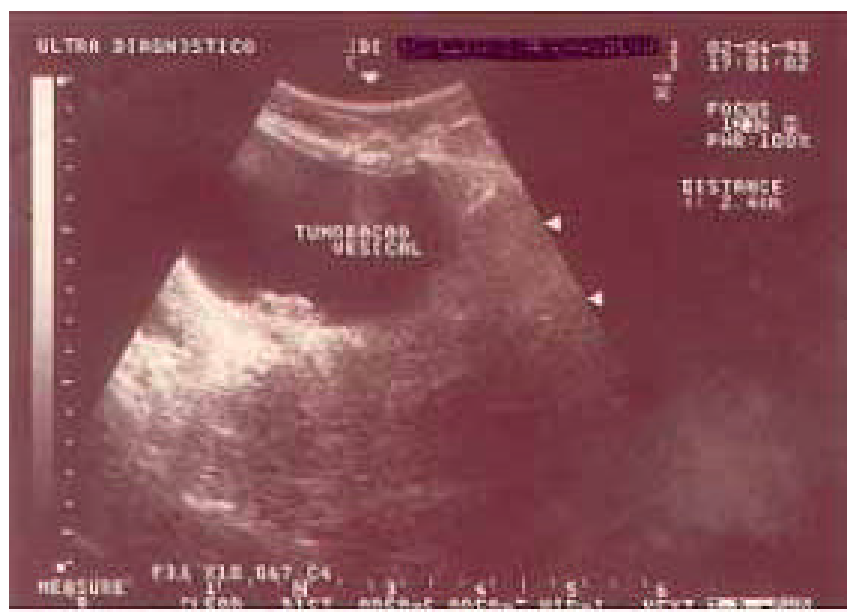

Figura 3 - Ultra-sonografia da bexiga evidenciando lesão vegetante da parede vesical com extensão intraluminal sugestiva de neoplasia.
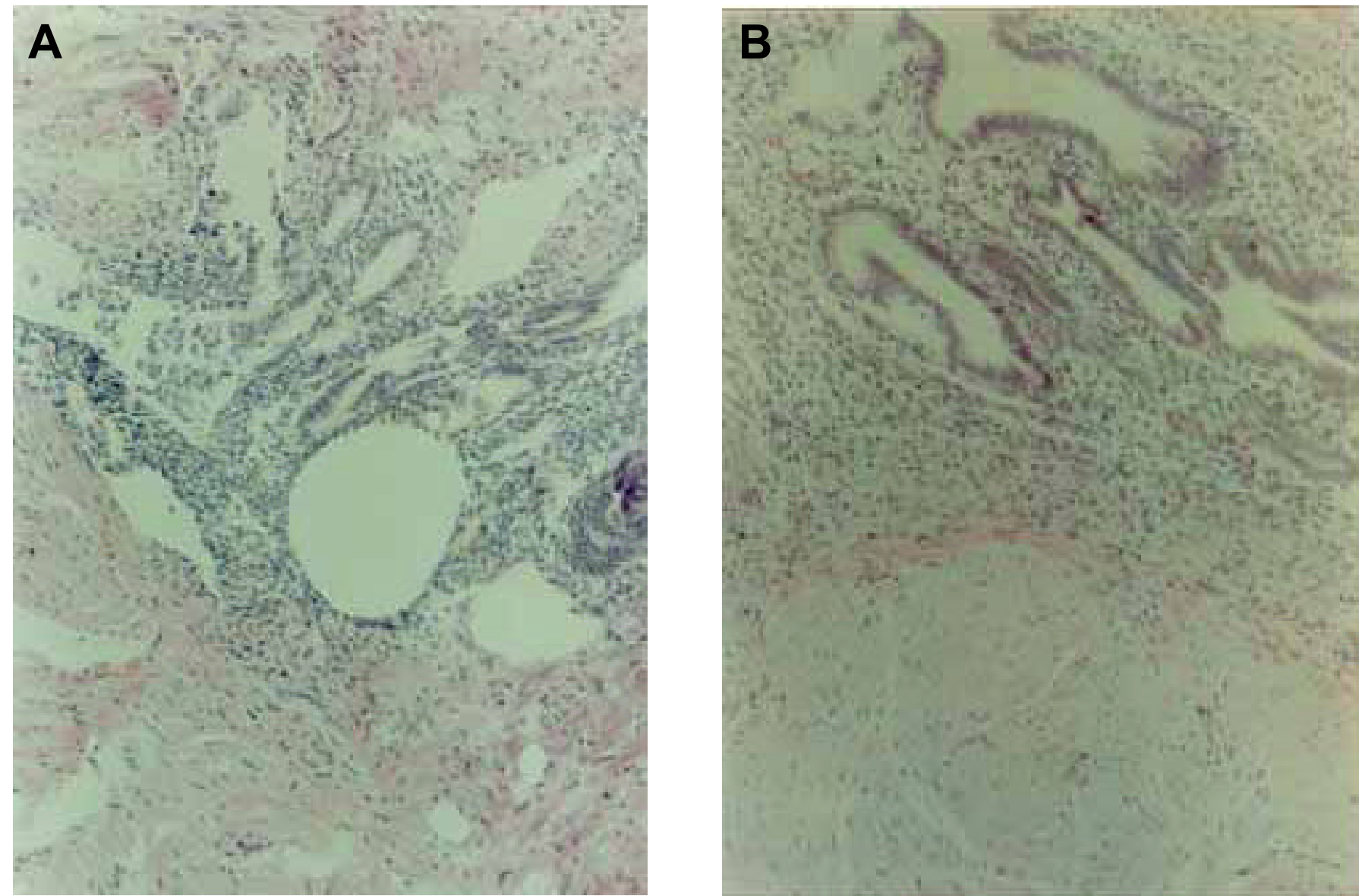

Figura 4 - A. Aspecto anátomo-patológico mostrando tecido endometrial invadindo a parede muscular da bexiga. B. Detalhe do epitélio colunar formando glândulas dilatadas do tecido endometrial. 
Paciente 3: Paciente de 41 anos com quadro de dor pélvica, contínua, incaracterística, que piorava após enchimento vesical e durante o coito, acompanhada de polaciúria, sem disúria ou hematúria. Durante investigação diagnóstica, há cinco anos, foi diagnosticada endometriose vesical por meio de laparoscopia e cistoscopia, sendo submetida a cistectomia parcial. Os sintomas recidivaram. A USG mostrou tumor retrovesical de $2 \mathrm{~cm}$ de extensão. Nova cistoscopia mostrou abaulamento do fundo vesical com mucosa vesical integra. Foi tratada com análogos de GnRH, melhorando dos sintomas e ocorrendo regressão da lesão retrovesical. Mantém-se em terapia hormonal e observação clínica, com tempo de seguimento de 1 ano, sem novas recidivas.

Paciente 4: Paciente de 35 anos com história de dor pélvica há mais de 6 meses associado a disúria e polaciúria. Recebeu antibioticoterapia por suposta infecção urinária de repetição, sem melhora. A ultra-sonografia revelou massa intravesical de $5 \mathrm{~cm}$ no fundo vesical. A cistoscopia prévia à ressecção endoscópica revelou lesão bocelada, azulada e com mucosa vesical integra. O exame anátomo-patológico da peça ressecada revelou endometriose vesical. Foi encaminhada ao grupo de ginecologia, que optou por utilizar análogo do GnRH por 3 meses. Encontra-se no $6^{\circ}$ mês de seguimento pós-operatório, com melhora dos sintomas e ausência de sinais de recidiva local.

\section{Discussão}

A faixa etária de nossas pacientes foi compativel com a de outros relatos, mostrando predominância dos 25 aos 40 anos.

O quadro clínico observado foi muito variado e depende do tamanho da lesão e do ciclo menstrual, o que também é compativel com outros relatos (Tabela 2) 1-5 $^{1-}$ O quadro clássico de sintomas irritativos vesicais, urocultura estéril, hematúria cíclica e tumor vesical em pacientes jovens nos obriga a pensar na possibilidade de endometriose vesical $^{1,6}$.

Tabela 2 - Freqüência dos sintomas em endometriose vesical ${ }^{1-5}$

\begin{tabular}{lc}
\hline Sintoma & Freqüência (\%) \\
\hline Desconforto suprapúbico & 78 \\
Hidronefrose/dor lombar & 14,2 \\
Tumor palpável & $39-45$ \\
Urgência/disúria & $71-86$ \\
Incontinência & 3,5 \\
Hematúria & $25-28$ \\
Piúria & 5 \\
Alterações menstruais & 42 \\
\hline
\end{tabular}

A USG pode mostrar a presença de massa extrinsica e contigua à bexiga ou invadindo a luz do órgão. Muitas vezes os achados são indistinguiveis de uma neoplasia vesical, mostrando imagem que se origina na parede e apresenta projeção intraluminal. Estes achados podem ser confirmados pela tomografia computadorizada, que permite observar a extensão e presença de lesões concomitantes.

A confirmação diagnóstica é realizada mediante uretrocistoscopia com biópsia, em que os principais achados são lesões superficiais violáceas com saída de secreção achocolatada, edema perilesional, mucosa vesical integra e, raramente, lesões ulcerativas com invasão de mucosa $^{2}$. Devemos lembrar que nem sempre as lesões típicas estão presentes, sendo que nestes casos devemos realizar biópsia profunda da parede vesical, pois biópsias superficiais trazem apenas mucosa com tecido insuficiente e freqüentemente resultam no diagnóstico histológico de cistite inespecífica.

Nestes casos está indicada a ressecção endoscópica, que permite a remoção de tecido adequado para diagnóstico, além de possibilitar a exérese total de lesões de pequenas dimensões. Eventualmente, a laparoscopia diagnóstica pode ser realizada em casos duvidosos ou quando existe necessidade de avaliar outros órgãos pélvicos ou realizar biópsias de lesões suspeitas.

Os achados anátomo-patológicos incluem desde lesões microscópicas sem invasão da serosa vesical até massas de grandes proporções com invasão muscular e da mucosa vesical. A histologia revela a presença de tecido glandular endometrial infiltrado entre as fibras musculares lisas da bexiga. As glândulas podem estar preenchidas por sangue ou material necrótico, refletindo as várias fases do ciclo menstrual. Raramente ocorre degeneração maligna, caracterizada por adenocarcinoma de origem endometrial ${ }^{3}$.

Existem várias opções de tratamento: as modalidades não-cirúrgicas incluem radioterapia e tratamento clinico com dietilestilbestrol, andrógenos, contraceptivos orais, danazol e análogos de GnRH, sendo que este último parece ser o mais eficiente. Os medicamentos agem modificando a resposta cíclica do endométrio aos hormônios femininos, causando regressão do tecido endometrial ${ }^{7-10}$. No nosso Serviço, as pacientes recebem acompanhamento da Disciplina de Ginecologia após a ressecção cirúrgica, optandose pelo tratamento com análogos de GnRH se a paciente tiver condições financeiras de arcar com o custo desta medicação. Caso contrário, opta-se por algum outro medicamento. O período de tratamento é variável de acordo com a resposta tera- 
pêutica, num período mínimo de 3 meses.

Alguns autores relatam que o tratamento cirúrgico parece trazer os melhores resultados, podendo ser realizado através de fulguração ou ressecção das lesões. Neste último caso, pode-se realizar ressecção da lesão por via endoscópica ou por meio de cistectomia parcial. Quando ocorre acometimento ureteral, opta-se por ressecção da lesão e cateterismo do ureter ou reimplante ureteral primário.

Stanley et al. ${ }^{9}$ apresentaram 10 casos de endometriose vesical, dos quais 7 foram submetidos à cistectomia parcial concomitante ao tratamento supressor de estrógenos; dentre estes, 6 pacientes evoluiram bem e uma das pacientes faleceu no pós-operatório. As 3 pacientes restantes foram tratadas apenas com supressão hormonal, apresentando também bons resultados ${ }^{9}$. Lavelle et al. ${ }^{6}$ trataram clinicamente uma paciente com obstrução ureteral por endometriose e obtiveram sucesso. Kistner ${ }^{2}$ demonstrou alivio dos sintomas com terapia progestagênica em mais de $80 \%$ das mulheres tratadas desta forma, entretanto Andrews ${ }^{4}$ acredita que esta terapêutica é paliativa e temporária. Devido às conseqüências da supressão estrogênica, Foster et al. ${ }^{1}$ acreditam que a melhor forma de abordar esta doença é pela cistectomia parcial seguida de tratamento adjuvante com hormônios, se houver implantes endometriais remanescentes.

Recentemente, Nezhat et al. ${ }^{11}$ descreveram 19 casos de sutura de bexiga, alguns após ressecção de endometriomas vesicais, por via laparoscópica. Estas pacientes foram mantidas com sonda vesical por 7 a 14 dias e apenas 1 mulher apresentou fistula vésico-vaginal que necessitou de correção. Todos os outros casos evoluíram satisfatoriamente e os autores concluiram que o tratamento laparoscópico é factível. Pela experiência adquirida nestes quatro casos e conhecimentos na revisão de literatura, podemos ressaltar alguns aspectos importantes: 1) o diagnóstico deve ser realizado por meio de biópsia profunda da parede vesical, geralmente conseguida pela ressecção com alça, pois biópsias superficiais podem revelar apenas cistite inespecífica; 2) a lesão vesical é manifestação secundária à doença multifocal e, em nossa opinião, deve sempre ser tratada com terapia hormonal, exceção feita a pacientes com lesão completamente ressecável e motivadas para engravidar; 3) a magnitude da massa que invade a bexiga varia de pequenas a grandes dimensões. O tamanho da lesão parece influenciar a resposta à terapia clínica exclusiva, pois, em nossa experiência, o único caso com lesão de grandes dimensões não teve resposta adequada em um seguimento a curto prazo; 4) o tratamento clínico isolado pode ser bem sucedido no controle sintomático e na regressão radiológica da lesão, especialmente se esta for de pequena dimensão; 5) o tratamento cirúrgico por meio da ressecção de lesões é a modalidade terapêutica mais eficaz para erradicar a massa e eliminar os sintomas locais urinários. Se o tratamento hormonal adjuvante não for empregado, pode haver recidiva dos sintomas e surgimento de novas lesões.

$\mathrm{O}$ tratamento de pacientes com endometriose do trato urinário é ainda motivo de discussão. Bons resultados foram obtidos com as várias formas terapêuticas e são defendidas por seus autores. O tratamento ideal deve ser baseado em suas particularidades como o tamanho da lesão, a gravidade dos sintomas, o desejo reprodutivo da paciente e a aderência ao tratamento.

\section{SUMMARY}

Purpose: urinary tract involvement by endometriosis is uncommon and the bladder is the most common site. We observed that clinical misdiagnosis of bladder cancer frequently is made. Because the disease is generally described in case reports there is not a consensual management. We present and discuss our experience of diagnostic and therapeutic issues.

Methods: retrospective analysis of urinary endometriosis slides of the Department of Pathology files was made. Medical charts and follow-up were reviewed in detail and interviews were performed during or after treatment.

Results: we describe four cases with cyclic disuria, abdominal mass, pelvic pain and imaging diagnosis of bladder tumor. Pathological specimens were obtained by endoscopic resection (3 cases) and laparoscopic biopsy (1 case). Therapeutic options were exclusive medical treatment or surgical removal with transurethral resection or partial cystectomy supplemented with adjuvant medication.

Conclusions: we review the clinical and therapeutic aspects of urinary tract endometriosis stressing that this is an important differential diagnosis of bladder cancer in reproductive women.

KEY WORDS: Endometriosis. Endoscopic surgery. Bladder: neoplasm.

\section{Referências}

1. Foster RS, Rink RC, Mulcahy JJ. Vesical endometriosis: medical or surgical treatment. Urology 1987; 29:64-5.

2. Kistner RW. The use of progestins in obstetrics and gynecology. Chicago: Year Book Medical Publishers; 1969. p.73. 
3. Abeshouse BS, Abeshouse G. Endometriosis of the urinary tract: a review of the literature and a report of four cases of vesical endometriosis. J Int Coll Surg 1960; 34:43-58.

4. Andrews WC. Medical versus surgical treatment of endometriosis. Clin Obstet Gynecol 1980; 23:917-24.

5. Arap Neto W, Lopes RN, Cury M, Montelatto NID, Arap S. Vesical endometriosis. Urology 1984; 24:271-4.

6. Lavelle KJ, Melman AW, Cleary RE. Ureteral obstruction owing to endometriosis: reversal with synthetic progestin. J Urol 1976; 116:665-6.

7. Price DT, Maloney KE, Ibrahim GK, Cundiff GW, Leder RA, Anderson EE. Vesical endometriosis: report of two cases and review of the literature. Urology 1996; 48:639-43.
8. Schwartzwald D, Mooppan UM, Ohm HK, Kim H. Endometriosis of the bladder. Urology 1992; 39:219-22.

9. Stanley KE Jr, Utz DC, Dockerty MB. Clinically significant endometriosis of the urinary tract. Surg Gynecol Obstet 1965; 120:491-9.

10.Vercellini P, Meschia M, De Giorgi O, Panazza S, Cortesi I, Crosignani PG. Bladder detrusor endometriosis: clinical and pathogenetic implications. J Urol 1996; 155:84-6.

11.Nezhat CH, Seidman DS, Nezhat F. Laparoscopic management of intentional and unintentional cystotomy. J Urol 1996, 156:1400-2. 